\title{
Electron Optics Design and Performance of a new Large-format Two-frame Framing Tube
}

Ching C. Lai

This article was submitted to the $25^{\text {th }}$ International Congress on High Speed Photography and Photonics, Beaune, France, September $29^{\text {th }}$ to October $4^{\text {th }}, 2002$

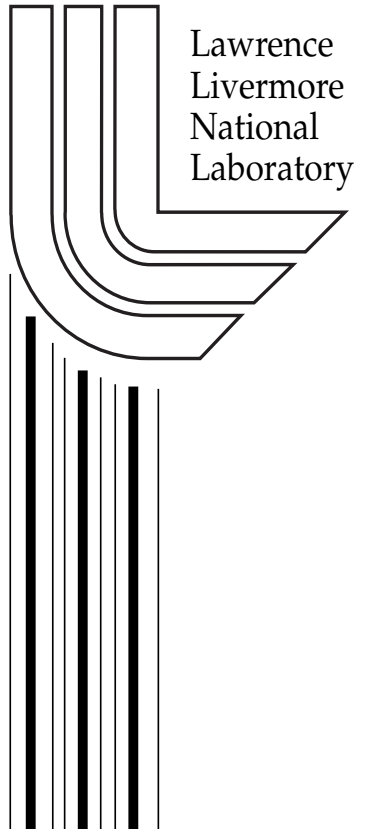

November $8^{\text {th }}, 2002$ 


\section{DISCLAIMER}

This document was prepared as an account of work sponsored by an agency of the United States Government. Neither the United States Government nor the University of California nor any of their employees, makes any warranty, express or implied, or assumes any legal liability or responsibility for the accuracy, completeness, or usefulness of any information, apparatus, product, or process disclosed, or represents that its use would not infringe privately owned rights. Reference herein to any specific commercial product, process, or service by trade name, trademark, manufacturer, or otherwise, does not necessarily constitute or imply its endorsement, recommendation, or favoring by the United States Government or the University of California. The views and opinions of authors expressed herein do not necessarily state or reflect those of the United States Government or the University of California, and shall not be used for advertising or product endorsement purposes.

This is a preprint of a paper intended for publication in a journal or proceedings. Since changes may be made before publication, this preprint is made available with the understanding that it will not be cited or reproduced without the permission of the author.

This report has been reproduced directly from the best available copy.

Available electronically at http://www.doc.gov/bridge

Available for a processing fee to U.S. Department of Energy

And its contractors in paper from

U.S. Department of Energy

Office of Scientific and Technical Information

P.O. Box 62

Oak Ridge, TN 37831-0062

Telephone: (865) 576-8401

Facsimile: (865) 576-5728

E-mail: reports@adonis.osti.gov

Available for the sale to the public from

U.S. Department of Commerce

National Technical Information Service

5285 Port Royal Road

Springfield, VA 22161

Telephone: (800) 553-6847

Facsimile: (703) 605-6900

E-mail: orders@ntis.fedworld.gov

Online ordering: http://www.ntis.gov/ordering.htm

OR

Lawrence Livermore National Laboratory

Technical Information Department's Digital Library

http://www.llnl.gov/tid/Library.html 


\title{
Electron optics design and performance of a new large-format two-frame framing tube*
}

\author{
Ching C. Lai \\ Lawrence Livermore National Laboratory, Livermore, California 94551-0808
}

\begin{abstract}
We have developed a framing tube with $80-\mathrm{mm}$ photocathode for capturing two frames in less than 100-ns onto a 50-mm phosphor screen. A proven electron optics trajectory code was used to design the tube for imaging fidelity over wide dynamic range. This code's full accounting of space charge effects is essential for its ability to simulate accurately the distributed photoelectronic trajectories from the entire large photocathode area. Our approach and guideline for designing the electron optics are described. Results of trajectory simulation and test measurement are reported. Substantial correlations between the code expectation and the measured results are observed on relative resolution and distortion of the frame images. This tube has been integrated into an active framing camera system for field application.
\end{abstract}

Keywords: electron optics design, computer modeling, trajectory analysis, framing tube, image converter

\section{INTRODUCTION}

A tube with large photocathode area is essential for sensitive imaging of an extended diffusing object. However, to our knowledge, all the available framing tubes are of rather small photocathode format, at $10 \times 10 \mathrm{~mm}^{2}$ or less. We thus initiate to develop a new framing tube with $80-\mathrm{mm}$ diameter photocathode for capturing two frames in as short as 100-ns apart onto a 50-mm diameter phosphor screen. A joint effort with tube manufacturer was carried out by LLNL designing the tube electron optics and the selected vendor (Photek of UK) building the tube. Here, we report the electron optics design as well as the relevant measurements on static parameters of tube performance.

\section{ELECTRON OPTICS DESIGN CODE}

The code program we employed is known as EGUN ${ }^{1}$ authored by Herrmannsfeldt and resided in a Cray2 main frame computer. It accepts a voltage boundary at $1 \mathrm{~mm} / \mathrm{mesh}$ scale with finer interpolation between mesh points and accounts fully the space charge effect. For framing tube design, the coordinate can be transformed from cylindrical symmetry in the imaging section to rectangular symmetry in the deflection section. At the end of each trajectory trace, the program yields detailed positional data at the termination surface of phosphor screen. Off-line processing of those data yields expected tube performance parameters including estimation of relative resolution and calculation of distortion. Over the years, this program has been used successfully by this author for numerous applications such as designing the LLNL/ITT streak tube ${ }^{2}$ and verifying the RCA streak tube ${ }^{3}$.

\section{APPROACHES FOR DESIGNING THE ELECTRON OPTICS}

Given the high detection sensitivity being a requisite for tube performance, the design allows no obstruction or barrier for the photoelectron path from photocathode to phosphor screen. No accelerating grid or mesh near the photocathode is incorporated. Neither is a mesh adopted for the anode aperture. The absence of an accelerating mesh does requires a strong field gradient to limit the space charge effect near the photocathode where the photoelectrons are slow with long dwell time. The large active photocathode area requires large focusing electrodes to generate a uniform field for minimizing both the distortion and the decaying resolution toward the edge. It becomes apparent that a set of large and multiple focusing electrodes in certain configuration are in order for the electron optics.

The sequence to arrive at an optimal tube configuration starts with the imaging section in front of the anode aperture then followed by the deflection region behind the aperture. Photocathode area and tube demagnification ratio are the key pre-determined parameters. Detailed geometry of all interior components and number of focusing electrodes are the variables to attain an overall optimized performance in relative resolution, distortion and dynamic range. Other

* This work was performed under the auspices of the U.S. Department of Energy by the University of California, Lawrence Livermore National Laboratory under contract No. W-7405-Eng-48. 
constraints are hardware component availability and fabricability. The result is indeed a fairly large tube $(11 \mathrm{~cm}$ diameter by $45 \mathrm{~cm}$ long) with three focusing electrodes, curved photocathode and screen. Figure 1 shows the finalized tube's interior boundary configuration and the optimized voltage setting for all focusing electrodes.

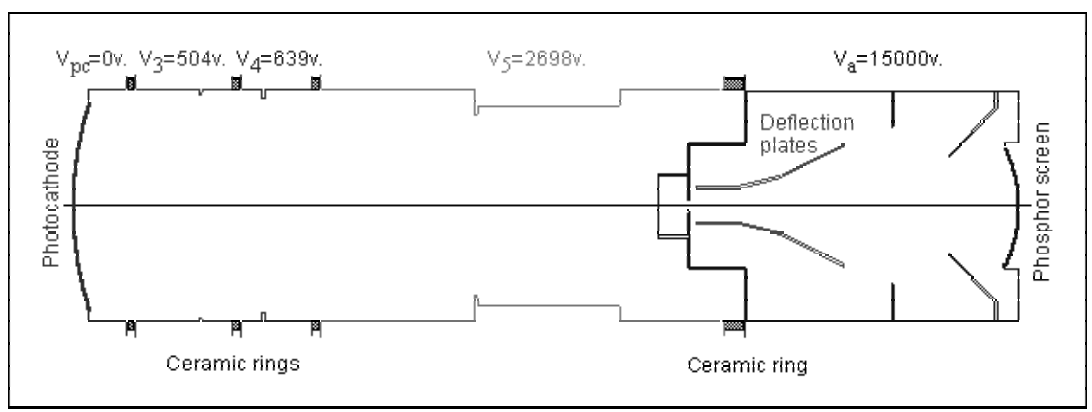

Figure1. Tube interior boundary configuration of the finalized electron optics design. It also strives for simplifying the tube fabrication.

For the photoelectron trajectory simulation, 11 radial locations distributed uniformly across the photocathode are selected as the starting image points for the trajectory traces. Three trajectories are launched from each image points with the central one being normal to and two side ones being $\pm 60^{\circ}$ to the photocathode surface. The current density of each trajectory beam is properly weighted such as to simulate a uniform photoelectron current density emitting from the entire photocathode area. Each side beam, the current density is assigned as having $1 / 3$ of that of its corresponding central beam. Figure 2 shows the typical plots of trajectory traces. For better visibility of the tray focusing variations, the scale of the vertical axis is more than seven times finer than that of the horizontal axis.
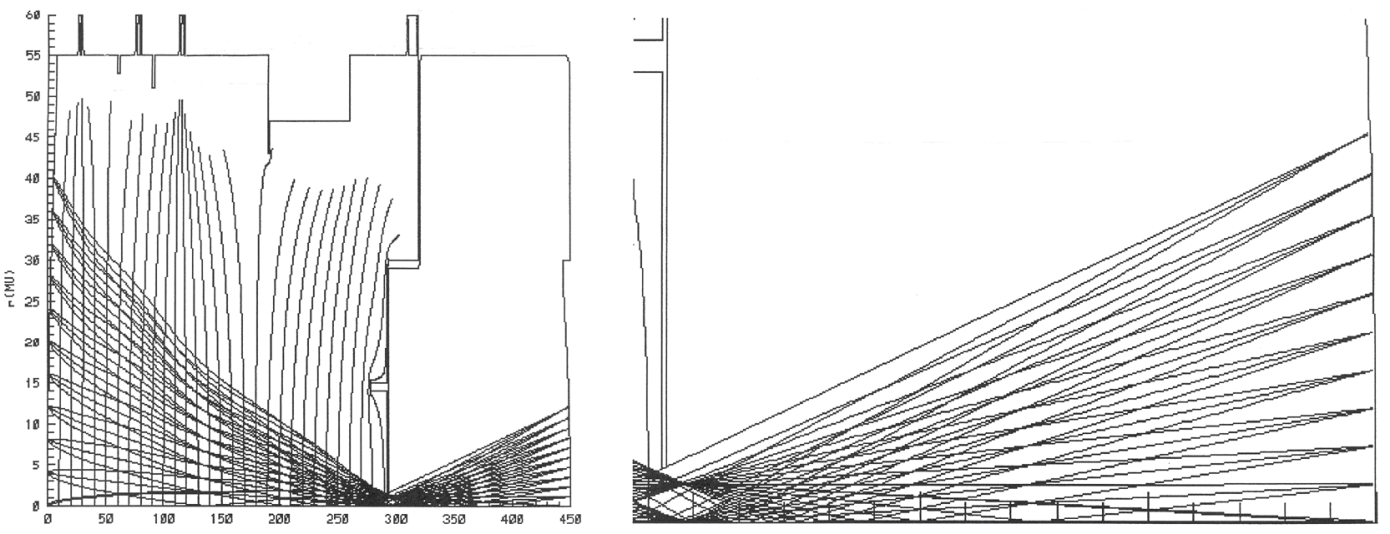

Figure 2. Typical trajectory traces plots generated by the code. On the right is an expanded version of the section behind the anode aperture. The coordinate scales are different between the two axes.

Positional spread of the 3-beam from a given image point at the screen defines its spot size. One half of the inverse of this spot size in $\mathrm{mm}$ yields an estimation of relative resolution in $1 \mathrm{p} / \mathrm{mm}$ for the image point. Note that this measure being an estimate at best is due to the fact that the realizable resolution results from a complex convolution of several factors including work function of photocathode material, angular distribution of photoelectron emission, phosphor material property, and others. However, this calculated resolution does provide the only measure that allows relative assessment of important tube performance parameters. Throughout the design process, the goal is to achieve a higher calculated resolution for every one of the 11 imaging points distributing over the entire image frame.

\subsection{Photoelectron Current and Dynamic Range}

As the current of emitting photoelectrons increases, the space charge effect among them gradually becomes significant in the form of degrading resolution over some portion of the image. The degraded resolution limits the acceptable dynamic range of incident intensity. The design goal is to attain high overall resolution at low current as well as to minimize the resolution degradation at high current. Figure 3 shows the calculated resolution of five trajectory runs at the total photoelectron currents ranging from $0.07 \mu$ a to $1.38 \mu$ a, i.e. over a nearly 2000 -to- 1 range. The tube voltage for all these runs is set at $14 \mathrm{kV}$. 


\subsection{Tube Voltage Effect}

At higher tube voltage, as the transit duration of photoelectron shortens, it reduces the adverse effect of space charge interaction. Resolution thus increases with tube voltage, especially under high photoelectron conditions. Figure 4 shows the calculated resolution at high current of $138 \mu$ a for a tube voltage setting ranging from at $7 \mathrm{kV}$ to $20 \mathrm{kV}$. The resolution improvement at higher tube voltage is clear and consistent. However, a tube operated at very high voltage may not be practical for use in humid environment. Furthermore, the deflection circuitry's ringing instability becomes more difficult to manage at higher voltage. A framing camera must maintain very narrow tolerance stability during the entire frame period, including particularly the time immediately adjacent to both sides of frame switching. It is a trade-off between practicality and ideal performance superiority. We settled at $15 \mathrm{kV}$ for the tube voltage.

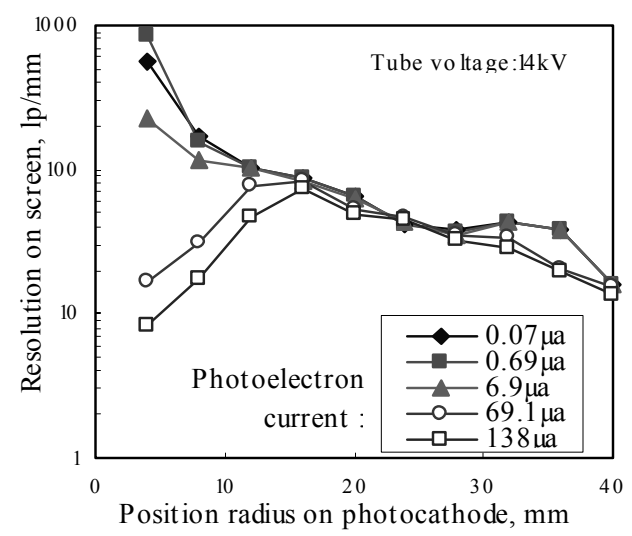

Figure 3. Calculated resolution variations for photoelectron current over a dynamic range of nearly 2000-to- 1 . The resolution degrades with photoelectron current.

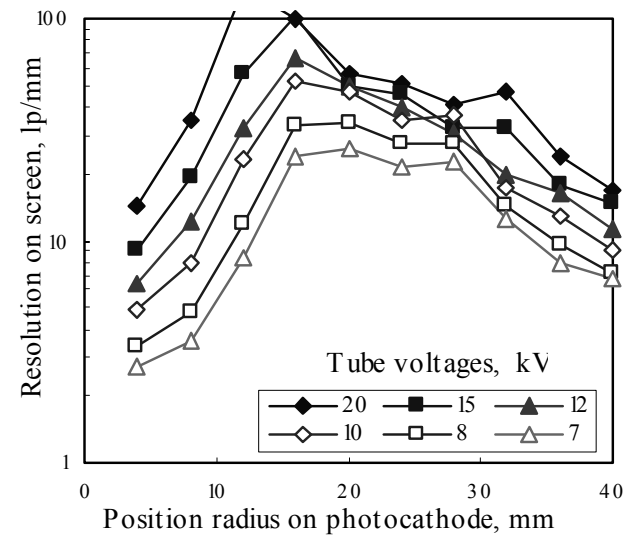

Figure 4. Calculated resolution at high photoelectron current of $138 \mu$ a for tube voltages from 7 to $20 \mathrm{kV}$. The resolution improves significantly with tube voltage.

\subsection{Design for the Framing Deflection Region}

For designing the framing deflection region, all the focusing-region trajectories are terminated at the anode aperture where no field gradient exists. The positional data in cylindrical coordinate are then transformed into corresponding rectangular coordinate. Additionally, a set of complemental mirror reflection trajectories are generated to fill the full space of both above and below the center line. Their current densities are properly transformed as well to simulate a uniform distribution. Since the EGUN program is not a complete 3-D code, the transformation does incur certain incompleteness into the simulation for particularly in the deflection region. Figure 5 shows the typical trajectory trace plots for the upper and the lower frame.
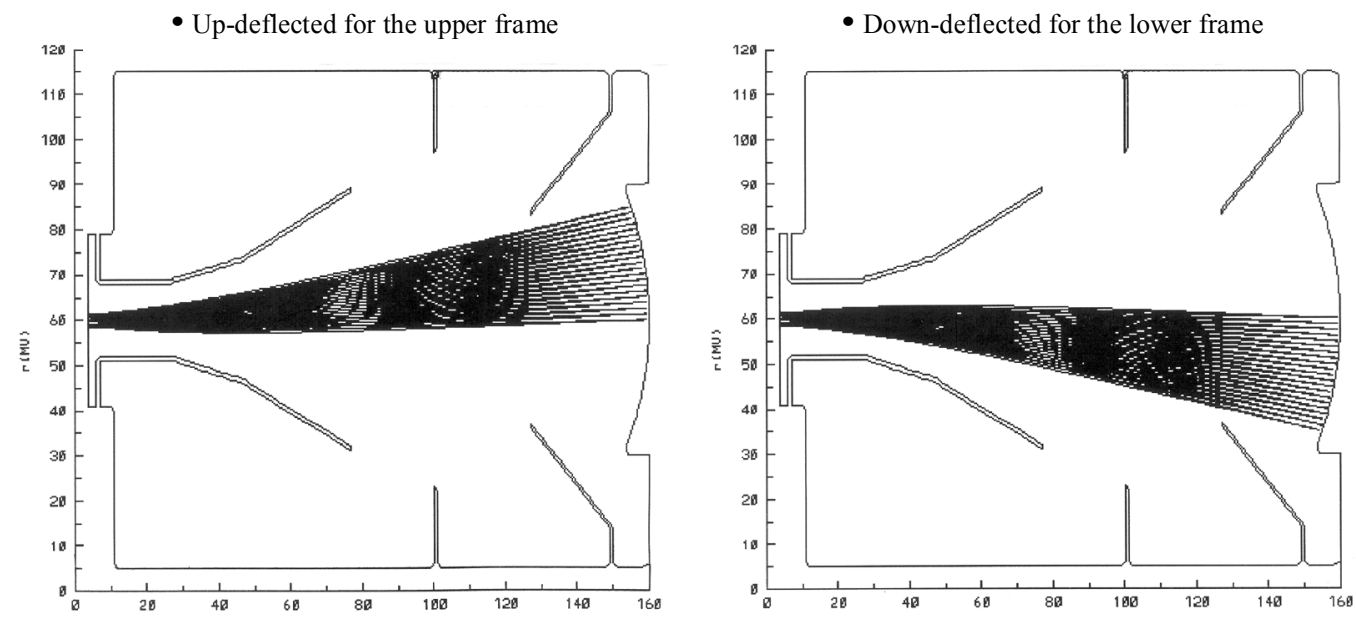

Figure 5. Typical plots of trajectory trances in the framing deflection region. The coordinate here is of retangular symmetry 
Here the design criteria are desired resolution, distortion, deflection sensitivity, and accommodation for beam parking with high extinction ratio before and after framing. The variables are mainly the geometry of deflection plates and parking pockets. Under the deflecting field, the resolution of frame images degrades substantially along the framing or temporal direction. The location and shape of the deflection plates affect the degradation magnitude. In general, wider plate separation lessens the degradation but at the expense of poorer deflection sensitivity. The deflection sensitivity of this tube is $13.1 \mathrm{~mm} / \mathrm{kV}$. Varying the focusing electrode voltages and changing the phosphor screen geometry can also reduce the degradation. A 3-D code would be helpful for detailed simulation on the effect of the deflection mechanism. The parking pockets are needed to ensure high extinction of unwanted signal or noise integration outside the temporal window of both frames. No gating mechanism is incorporated in this tube for blocking the exposure during the frame switching transient of about 100ns, or during the transit shifting between pocket and frame locations.

\section{COMPARISON WITH MEASURED RESULTS OF TUBE PERFORMANCE}

The first and only tube was successfully built by Photek of United Kingdom as per the electron optics design. Shown in Figure 6 , the tube assembly is large $(175 \mathrm{~mm}$ diameter by $474 \mathrm{~mm}$ long) and robustly packaged. It is RTV potted within mu-metal casing with stainless steel flanges at both ends. After the tube was fabricated a resistance leakage path at $635 \mathrm{k} \Omega$ between the first and the second focusing electrode was identified. It results in severe image clipping if operated under the simulation designed set of electrode voltages. Figure 7 illustrates this leakage path and the designed voltages. However, with some adjustments, an optimized set of voltages was reached to produce acceptable unclipped images. The optimized set is shown within the parentheses in Figure 7. It is hence employed for operating the tube. In the following, trajectory results shall be presented along with the measured result of corresponding performance parameters.

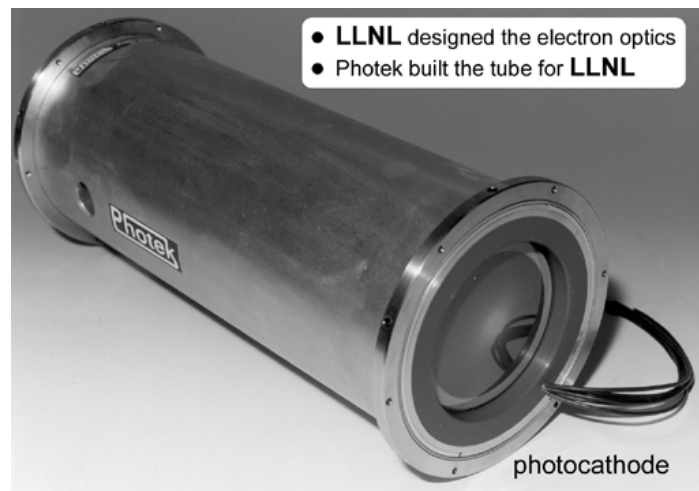

Figure 6. This tube assembly is large in size at $175 \mathrm{~mm}$ diameter by $474 \mathrm{~mm}$ length and robustly packaged.

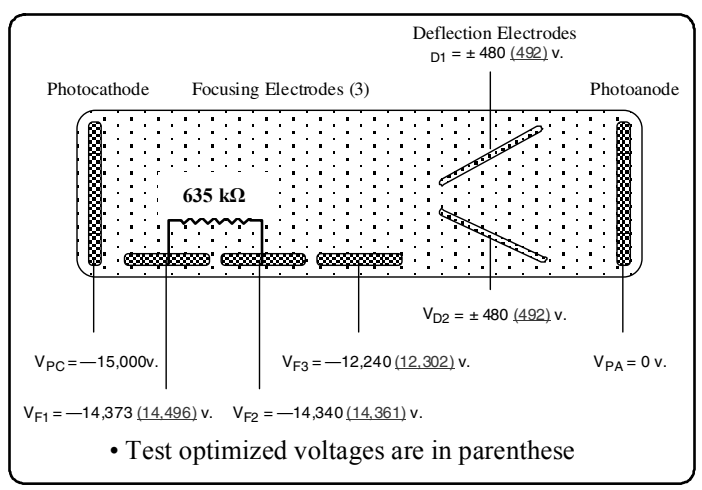

Figure 7. Trajectory designed voltages are listed with test optimized setting to accommodate resistance leakage blemish.

\subsection{Relative Resolution of Frame Images}

As stated earlier, due to influence of the deflecting field, the resolution of frame image is adversely affected. Figure 8 shows the calculated resolution along the framing axis for the deflected frames as well as for the static image without deflection under the best focusing electrode setting. For measuring the tube performance, a USAF test target attached to the center of photocathode under flat field illumination was utilized for the resolution evaluation. Figure 10 shows the up-deflected frame image of the target as acquired by a video CCD camera. Among the images acquired, the two images at deflected frame locations exhibit noticeably poorer resolution than the un-deflected one. This is expected and can be improved somewhat by re-adjusting the electrode voltage setting specifically for best focusing of the deflected images in actual framing mode operation. The USAF target images were analyzed along the framing axis in terms of their contrast transfer function (CTF). Figure 9 is the CTF plots for these three frames. It exhibits substantially lower CTF for the deflected frames. The correlation between the trajectory calculation in Figure 8 and the test measurement in Figure 9 is obvious.

\subsection{Normalized Distortion}

In trajectory simulation, evaluation of image distortion depends only on positional data and thus is not quantified in relative sense as for the case of resolution. Furthermore, the distortion is based on the positional data of the central beam 
of each 3-beam image point. Any deflecting field effect is expected to be more gradual and less severe than that on the resolution which is based on the relative positional spread of the 3 beams at each image point. The distortion reported henceforth is evaluated in terms of normalized percentage defined as distortion deviation divided by image diameter, i.e. $25 \mathrm{~mm}$ for this tube. Figure 12 shows the calculated distortion for deflected and un-deflected frame along the framing axis. It exhibits symmetrical variation for the no-deflection frame and asymmetrical skewness in opposite directions for the deflected frames. This is expected in light of the nonuniform deflection field influence along the framing direction.

For distortion measurements, a test pattern composed of an $80 \mathrm{~mm}$ diameter circle with two diametrally perpendicular heavy lines and fine grid lines of $2.5 \mathrm{~mm}$ separation was attached to the photocathode. Figure 11 is the acquired image of an up-deflected frame on the phosphor screen. A certain degree of distortion is visible near the edge. Also noticeable is the slight clipping at the lower left corner of the image. It is the residue of image clipping caused by the resistance leakage blemish of this first and only tube ever made to date. Detailed measurement of the grid line crossover point locations provides data base for quantitative distortion evaluation. Figure 12 shows measured distortion plots for all three frames along the framing axis. It exhibits a certain overall resemblance in the distortion variation trend across the image to that of the trajectory results in Figure 11. The magnitude of the measured distortion is about 2.5 times that of the trajectory calculation. This degree of discrepancy is not unexpected in view of all the intrinsic measurement uncertainties and the leakage blemish. The trajectory and the measured distortion profiles in Figure 12 and 13 are profoundly agreeable.

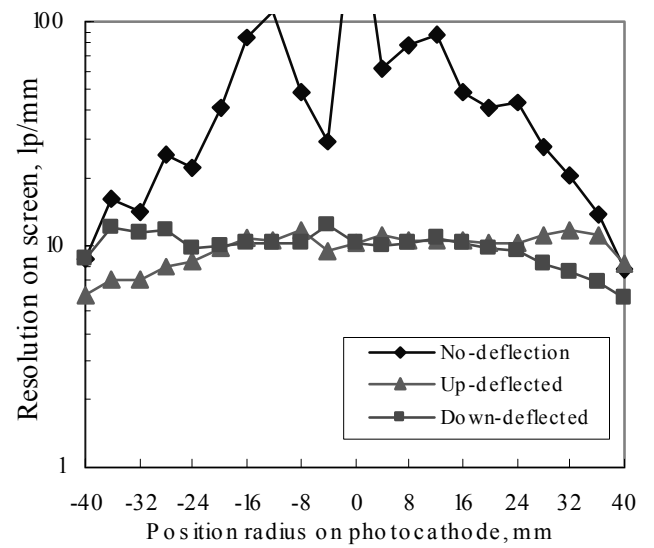

Figure 8. Calculated resolution degrades substantially in framing mode.

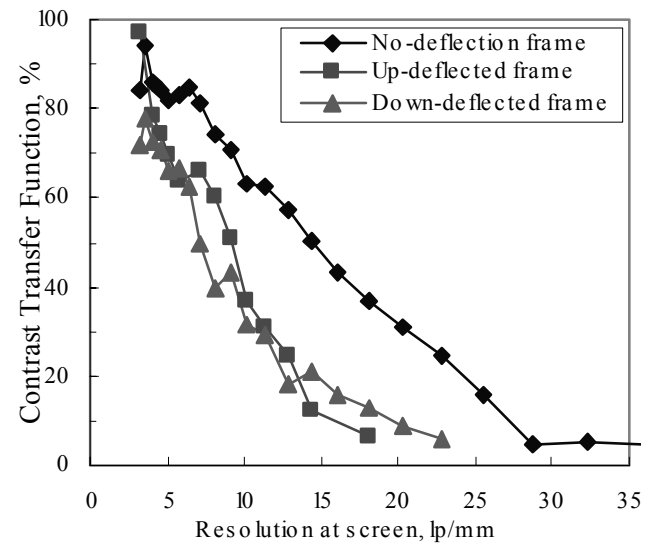

Figure 9. The measured CTF also shows degradation along the framing direction for the frame images.

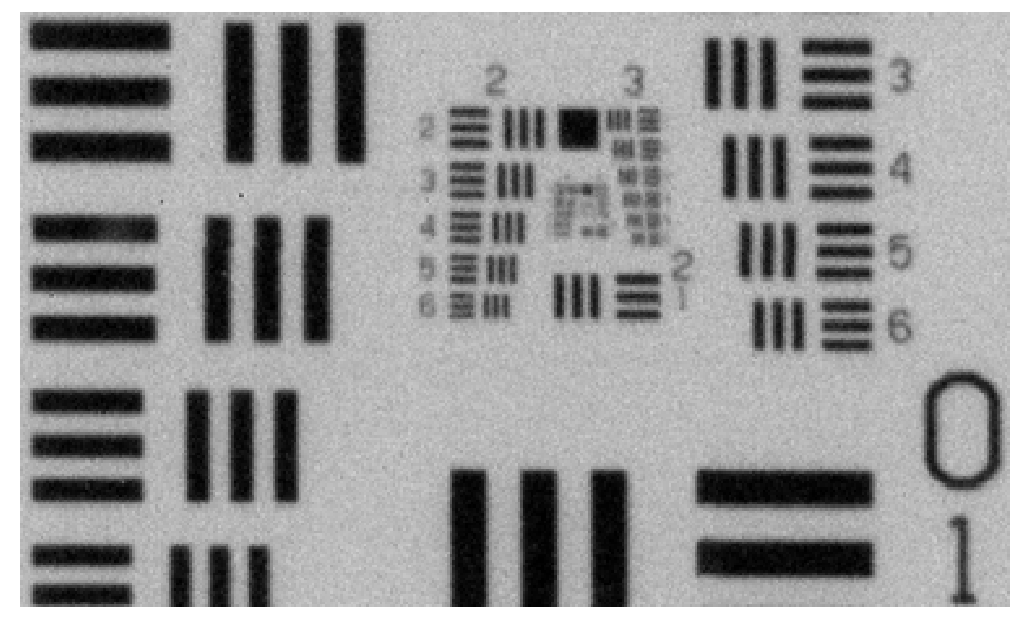

Figure 10. An up-deflected frame image of USAF target attached to the photocathode. This phosphor screen image is captured by a video CCD camera.

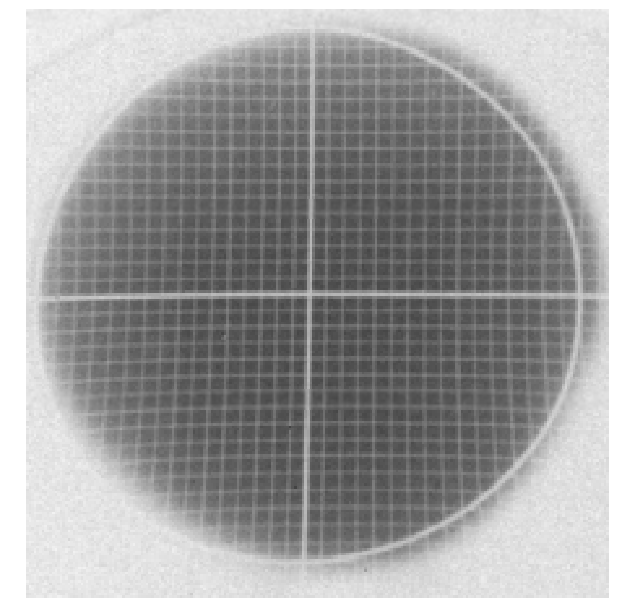

Figure 11. An up-deflected frame image of square grid pattern covering the entire photocathode area. 


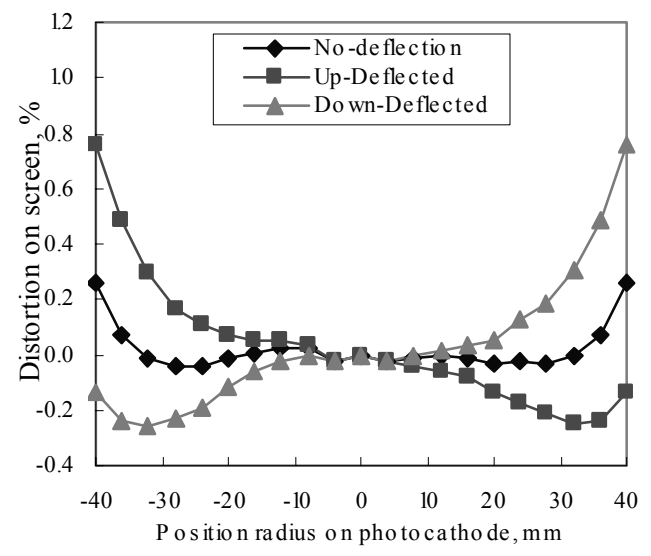

Figure 12. Trajectory calculated distortion along the framing axis.

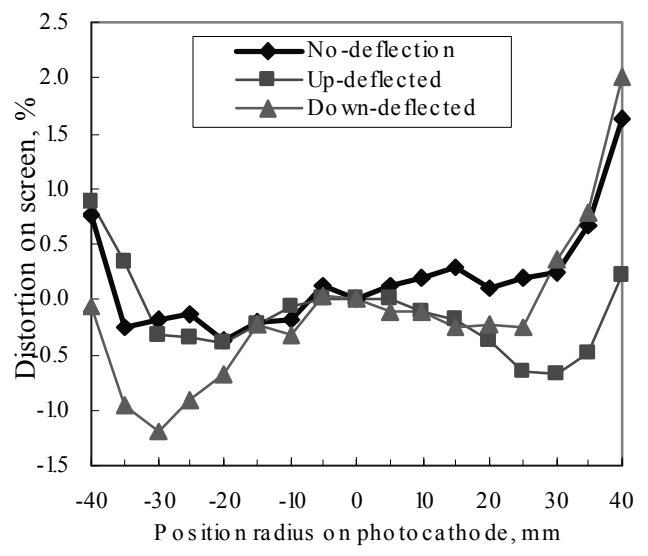

Figure 13. Measured distortion along the framing axis.

\section{CONCLUDING REMARKS}

Our electron optics design effort has resulted in a functional framing tube with satisfactory performance. The framing tube with such a large photocathode area is uniquely capable and suitable for sensitive high speed frame imaging of any extended diffusing source. Correlations between the trajectory expectation and the measurement result are substantial notwithstanding the resistance leakage blemish of the tube. The electron optics code program EGUN has further demonstrated its functionality as a rather reliable design tool for high speed framing tube. The tube reported here has been integrated with other components into an active framing camera system for use in our on-going field applications.

\section{ACKNOWLEDGEMENTS}

Portion of the test data were obtained during a pre-delivery evaluation at Photek and confirmed later at LLNL. Photek (M. Ingle, I. Philips, S. Mays and others) participated actively for the testing and provided the tube photo.

\section{REFERENCES}

1. W. B. Herrmannsfeldt, "EGUN- An Electron Optics and Gun Design Program", SLAC-331, UC-28, Stanford Linear Accelerator Center, Stanford University, Stanford, California, 1988

2. Ching C. Lai, "Design and Development of a New Streak Tube", LLNL EE Technical Review, UCRL-50025-861, UCAR-10159, Lawrence Livermore National Laboratory, Livermore, California, 1986

3. C. C. Lai, L. B. Olk, and R. D. Lear, "Photoelectronic Trajectory Anatomy of RCA-C73435 Image Converter and Its Performance," Proceeding 253 of IEE Conference on Photoelectronic Imaging, London, England, 1985 